\title{
EMITTANCE GROWTH IN RHIC DURING INJECTION
}

\author{
W. FISCHER *, W.W. MACKAY, S. PEGGS and J. WEI \\ Brookhaven National Laboratory, Upton, NY 11973, USA
}

(Received in final form 5 December 1996)

\begin{abstract}
Various processes will lead to emittance growth in RHIC during injection. The effects of steering errors, optical mismatch, space charge and intrabeam scattering are estimated. The transverse and longitudinal emittance growths are computed for protons and fully stripped gold ions.
\end{abstract}

Keywords: Emittance growth; Injection

\section{INTRODUCTION}

Emittance growth at any energy in the accelerator chain ultimately reduces the luminosity of a storage ring. In addition, when the transverse emittances exceed the dynamic or physical aperture, or when the longitudinal emittance exceeds the bucket admittance, the luminosity is further reduced due to particle loss.

The effects of magnetic multipole errors, tune modulation and space charge tune shift on the RHIC dynamic aperture at injection energy are presented elsewhere ${ }^{1,2}$. Collective instabilities, which can also lead to emittance growth, are treated in the collective instability reports ${ }^{3}$. Those effects are not addressed in this article. Here we investigate emittance growth caused by steering errors, optical mismatch, space charge and intrabeam scattering. For those effects formulae are given and estimates are made for both transverse and

\footnotetext{
* Corresponding author. Tel.: 001-516-344 5452. Fax: 001-516-344 5729. E-mail: wfischer@bnl.gov.
} 
longitudinal emittance growth. Protons and fully stripped gold ions are considered.

In Table I the beam parameters at injection are given for gold ions and protons. Table II contains the transverse lattice functions which are the same for both proton and gold operation. In Table III the longitudinal lattice parameters are shown. They are different for protons and gold ions. The data in Tables I-III are taken from the collective instability reports ${ }^{3}$.

TABLE I Beam parameters at injection

\begin{tabular}{lcccc}
\hline Parameter & Symbol & Unit & $\mathrm{Au}^{79+}$ & $\mathrm{p}^{+}$ \\
\hline Atomic number & $A$ & 1 & 79 & 1 \\
Mass number & $Z$ & 1 & 197 & 1 \\
Nominal bunch intensity & $N_{\mathrm{b}}$ & 1 & $10^{9}$ & $10^{11}$ \\
Number of bunches & $N$ & 1 & 60 & 60 \\
Kinetic energy & $E$ & $\mathrm{GeV} / \mathrm{u}$ & 10.8 & 28.3 \\
Relativistic gamma & $\gamma$ & 1 & 12.6 & 31.2 \\
Transverse rms emittance & $\epsilon_{x, y}$ & $\mu \mathrm{m}$ & 0.132 & 0.080 \\
Longitudinal rms emittance & $\epsilon_{s}$ & $\mathrm{~mm}$ & 0.261 & 0.158 \\
rms bunch length & $\sigma_{s}$ & $\mathrm{~m}$ & 0.774 & 0.384 \\
rms momentum spread & $\sigma_{p}$ & $10^{-3}$ & 0.351 & 0.411 \\
Filling time & $t$ & $\mathrm{~s}$ & 114 & 30 \\
\hline
\end{tabular}

TABLE II Transverse lattice functions at injection (identical for $\mathrm{Au}^{79+}$ and $\mathrm{p}^{+}$)

\begin{tabular}{lccccccc}
\hline Parameter & Symbol & Unit & \multicolumn{2}{c}{ Horizontal } & & \multicolumn{2}{c}{ Vertical } \\
\cline { 7 - 8 } \cline { 7 - 8 } & & & $\begin{array}{c}\text { Injection } \\
\text { kicker }\end{array}$ & Average & & $\begin{array}{c}\text { Injection } \\
\text { kicker }\end{array}$ & Average \\
\hline Beta & $\beta_{x, y}$ & $\mathrm{~m}$ & 15.6 & 23.91 & & 34.5 & 23.4 \\
Alpha & $\alpha_{x, y}$ & 1 & 0.938 & -0.225 & & -1.82 & -0.228 \\
Dispersion & $D_{x, y}$ & $\mathrm{~m}$ & 1.029 & 0.602 & & 0.0 & 0.0 \\
$\begin{array}{l}\text { Dispersion } \\
\text { slope }\end{array}$ & $D_{x, y}^{\prime}$ & 1 & -0.0704 & 0.0046 & & 0.0 & 0.0 \\
\hline
\end{tabular}

TABLE III Longitudinal lattice functions at injection

\begin{tabular}{lcccc}
\hline Parameter & Symbol & Unit & $\mathrm{Au}^{79+}$ & $\mathrm{p}^{+}$ \\
\hline Beta & $\beta_{s}$ & $\mathrm{~m}$ & 2268 & 934 \\
Momentum compaction & $\alpha$ & $10^{-3}$ & 1.91 & 1.91 \\
Transition gamma & $\gamma_{\mathrm{tr}}$ & 1 & 22.89 & 22.89 \\
Harmonic number & $h$ & 1 & 360 & 360 \\
Slippage factor & $\eta$ & $10^{-3}$ & -4.39 & +8.81 \\
\hline
\end{tabular}


Throughout this article $x$ denotes the horizontal, $y$ the vertical and $s$ the longitudinal coordinate. Further notation is given in Tables IIII. All emittances are quoted unnormalized by their rms value:

$$
\epsilon_{x, y}=\frac{\sigma_{x, y}^{2}}{\beta_{x, y}} \quad \text { and } \quad \epsilon_{s}=\frac{\sigma_{s}^{2}}{\beta_{s}}=\beta_{s} \sigma_{p}^{2}=\sigma_{s} \sigma_{p}
$$

\section{STEERING ERRORS}

The vertical emittance growth due to a steering error at injection is

$$
\frac{\Delta \epsilon_{y}}{\epsilon_{y}}=\frac{1}{2 \epsilon_{y} \beta_{y}}\left[(\Delta y)^{2}+\left(\alpha_{y} \Delta y+\beta_{y} \Delta y^{\prime}\right)^{2}\right]
$$

and a similar equation holds for the horizontal emittance growth. Assuming an error of $10^{-4}$ in the horizontal bending strength of the injection septum, the horizontal slope error is approximately $\Delta x^{\prime}=3.8 \mu \mathrm{rad}$.

The expected error of the vertical injection kicker is $13 \mu \mathrm{rad}$ (cf. the collective instability reports ${ }^{3}$ ). Position errors are expected to be less than $1 \mathrm{~mm}$. These numbers lead to relative emittance growths of

$$
\left(\frac{\Delta \epsilon_{x}}{\epsilon_{x}}\right)_{\mathrm{Au}}=0.48 \text { and }\left(\frac{\Delta \epsilon_{x}}{\epsilon_{x}}\right)_{\mathrm{p}}=0.80
$$

in the horizontal plane, and

$$
\left(\frac{\Delta \epsilon_{y}}{\epsilon_{y}}\right)_{\mathrm{Au}}=0.68 \text { and }\left(\frac{\Delta \epsilon_{y}}{\epsilon_{y}}\right)_{\mathrm{p}}=1.12
$$

in the vertical plane. To keep the emittance growth below $10 \%$, a transverse damper system will be installed ${ }^{3}$. Its damping time of $1.3 \mathrm{~ms}$ (100 turns) is sufficiently small compared to the decoherence time.

Systematic errors can be corrected in principle, but a change of the bending strength during bunch passage will always lead to an incoherent emittance growth. From all pulsed elements only the 
injection kicker is expected to show such noise. Imposing again a limit of $10 \%$ on the emittance growth an error in the kick strength of about $20 \mu \mathrm{rad}(50 \%$ larger than the systematic error) follows from Eq. (2).

The growth of the longitudinal emittance can be computed as

$$
\frac{\Delta \epsilon_{s}}{\epsilon_{s}}=\frac{1}{2 \epsilon_{s}}\left(\beta_{s}\left(\frac{\Delta p}{p}\right)^{2}+\frac{1}{\beta_{s}}(\Delta s)^{2}\right) .
$$

With the available technology the phase error and thereby $\Delta s$ can be neglected compared to the momentum error. The expected momentum error is $\Delta p / p=10^{-4}$ and one obtains

$$
\left(\frac{\Delta \epsilon_{s}}{\epsilon_{s}}\right)_{\mathrm{Au}}=4.3 \% \text { and }\left(\frac{\Delta \epsilon_{s}}{\epsilon_{s}}\right)_{\mathrm{p}}=2.9 \% .
$$

A longitudinal damper system is expected to reduce the longitudinal emittance growth by a factor of up to 100 (corresponding to a momentum error $\Delta p / p=10^{-5}$ ). Its limitation is the time resolution of the wall current monitor.

\section{OPTICAL MISMATCH}

For mismatched lattice functions $(\alpha, \beta)$ the emittance growth is ${ }^{4,5}$

$$
\frac{\Delta \epsilon}{\epsilon}=\frac{1}{2}\left[\frac{\beta_{1}}{\beta_{2}}+\left(\alpha_{1}-\alpha_{2} \frac{\beta_{1}}{\beta_{2}}\right)^{2} \frac{\beta_{2}}{\beta_{1}}+\frac{\beta_{2}}{\beta_{1}}\right]-1,
$$

where subscript 1 denotes values in the ring and subscript 2 values of the transfer line. Measurements performed during the AGS-to-RHIC transfer line commissioning indicate that lattice functions are known with $10 \%$ precision $^{6}$. This leads to

$$
\frac{\Delta \epsilon_{x}}{\epsilon_{x}}=2 \% \quad \text { and } \quad \frac{\Delta \epsilon_{y}}{\epsilon_{y}}=4 \%,
$$

which is the same for gold ions and protons. 
The effect of a dispersion mismatch is similar to a steering error (cf. Eq. (2)). The horizontal emittance growth can be computed as ${ }^{5}$

$$
\frac{\Delta \epsilon_{x}}{\epsilon_{x}}=\frac{1}{2 \epsilon_{x} \beta_{x}}\left[\left(\Delta D_{x}\right)^{2}+\left(\alpha_{x} \Delta D_{x}+\beta_{x} \Delta D_{x}^{\prime}\right)^{2}\right] \sigma_{p}^{2}
$$

and a similar equation holds for the vertical plane. Assuming a $10 \%$ dispersion mismatch in the horizontal plane the emittance growths become

$$
\left(\frac{\Delta \epsilon_{x}}{\epsilon_{x}}\right)_{\mathrm{Au}}=0.03 \% \quad \text { and } \quad\left(\frac{\Delta \epsilon_{x}}{\epsilon_{x}}\right)_{\mathrm{p}}=0.06 \%
$$

Since the injection kicker bends the incoming beam vertically there is a systematic vertical dispersion mismatch (with $\Delta D_{y}=0.088 \mathrm{~m}$ and $\left.\Delta D_{y}^{\prime}=-0.0036\right)$ which results in

$$
\left(\frac{\Delta \epsilon_{y}}{\epsilon_{y}}\right)_{\mathrm{Au}}=0.03 \% \quad \text { and } \quad\left(\frac{\Delta \epsilon_{y}}{\epsilon_{y}}\right)_{\mathrm{p}}=0.06 \%
$$

The longitudinal emittance growth due to mismatch in the RF system is

$$
\frac{\Delta \epsilon_{s}}{\epsilon_{s}}=\frac{\Delta \beta_{s}}{\beta_{s}}
$$

Experience shows that the RF voltage is known within 5-20\% precision. Since the synchrotron tune is proportional to the square root of the $\mathrm{RF}$ voltage $\left(Q_{s} \propto \sqrt{\hat{V}}\right)$, the synchrotron tune is known with $2.5-10 \%$ precision and with ${ }^{8}$

$$
\beta_{s}=\frac{C}{2 \pi} \frac{|\eta|}{Q_{s}}
$$

$C$ being the circumference ( $3833.845 \mathrm{~m}$ for RHIC), the longitudinal beta function is also known within $2.5-10 \%$. The expected emittance growth is then

$$
\frac{\Delta \epsilon_{s}}{\epsilon_{s}}=2.5-10 \%
$$




\section{SPACE CHARGE}

The transverse envelope equations for a Gaussian beam can be approximated by $^{7}$

$$
\begin{aligned}
& \sigma_{x}^{\prime \prime}+K_{x} \sigma_{x}-\frac{\epsilon_{x}^{2}}{\sigma_{x}^{3}}=\frac{\xi}{2\left(\sigma_{x}+\sigma_{y}\right)}, \\
& \sigma_{y}^{\prime \prime}+K_{y} \sigma_{y}-\frac{\epsilon_{x}^{2}}{\sigma_{x}^{3}}=\frac{\xi}{2\left(\sigma_{x}+\sigma_{y}\right)} .
\end{aligned}
$$

The space charge parameter $\xi$ reaches its maximum at the bunch center where it is

$$
\xi_{\max }=\frac{4 Z^{2} r_{0} \lambda}{A B \beta^{2} \gamma^{3}}
$$

$r_{0}$ is the classical proton radius:

$$
r_{0}=\frac{1}{4 \pi \epsilon_{0}} \frac{e^{2}}{m_{0} c^{2}}=1.5348 \cdot 10^{-18} \mathrm{~m},
$$

where $m_{0}$ denotes the proton rest mass and $c$ the speed of light;

$$
\lambda=\frac{N N_{\mathrm{b}}}{C} \quad \text { and } \quad B=\frac{\sqrt{2 \pi} N \sigma_{s}}{C}
$$

are the average longitudinal particle density and the bunching factor (ratio of the average longitudinal particle density to the maximum), respectively. For gold and protons one obtains

$$
\xi_{\max , A u}=5 \cdot 10^{-11} \text { and } \xi_{\max , \mathrm{p}}=2 \cdot 10^{-11} .
$$

Space charge leads to defocusing forces about $10^{5}$ times weaker than the external focusing provided by quadrupoles $\left(k=0.09 \mathrm{~m}^{-2}\right)$. This perturbation is in the order of the quadrupole strength ripple. Therefore, no emittance growth is expected from space charge 
effects. However, the Laslett tune shifts

$$
\Delta Q_{x, y}=-\frac{Z^{2} r_{o} C N_{\mathrm{b}}}{2(2 \pi)^{3 / 2} A \beta^{2} \gamma^{3} \epsilon_{x, y} \sigma_{s}}=-\frac{\xi_{\max } C}{16 \pi \epsilon_{x, y}}
$$

come out to be

$$
\Delta Q_{x, y ; \mathrm{Au}}=-0.029 \text { and } \Delta Q_{x, y ; \mathrm{p}}=-0.020
$$

which is about 10 times larger than the tune shift caused by nonlinear magnetic fields. The large tune shift is likely to reduce the dynamic aperture ${ }^{2}$.

\section{INTRABEAM SCATTERING}

The evolution of the rms beam size due to intrabeam scattering can be approximately described by ${ }^{9}$

$$
\left[\begin{array}{c}
\frac{1}{\sigma_{x}} \frac{\mathrm{d} \sigma_{x}}{\mathrm{~d} t} \\
\frac{1}{\sigma_{y}} \frac{\mathrm{d} \sigma_{y}}{\mathrm{~d} t} \\
\frac{1}{\sigma_{p}} \frac{\mathrm{d} \sigma_{p}}{\mathrm{~d} t}
\end{array}\right]=\frac{Z^{4} N_{\mathrm{b}}}{A^{2}} \frac{r_{0}^{2} L_{c}(\beta c)}{8 \pi(\beta \gamma)^{4} \epsilon_{x} \epsilon_{y} \epsilon_{s}} F(\chi)\left[\begin{array}{c}
-a^{2} / 2+d^{2} \\
-b^{2} / 2 \\
n_{\mathrm{b}}\left(1-d^{2}\right)
\end{array}\right] .
$$

For bunched beams $N_{\mathrm{b}}$ is the number of particles per bunch and $n_{\mathrm{b}}=1$, for unbunched beams $N_{\mathrm{b}}$ is the total particle number and $n_{\mathrm{b}}=2$. $L_{c}$ is a form factor which is approximately 20 . If vertical dispersion can be neglected, one has

$$
\begin{aligned}
& d=\frac{D_{x} \sigma_{p}}{\left(\sigma_{x}^{2}+D_{x}^{2} \sigma_{p}^{2}\right)^{1 / 2}}, \quad a=\frac{\beta_{x} d}{D_{x} \gamma}, \\
& b=\frac{\beta_{y} \sigma_{x}}{\beta_{x} \sigma_{y}} a \text { and } \quad \chi=\frac{a^{2}+b^{2}}{2} .
\end{aligned}
$$


The function $F(\chi)$ is defined by

$$
F(\chi)=\frac{(1+2 \chi) I(\chi)-3}{1-\chi}
$$

with

$$
I(\chi)= \begin{cases}\frac{1}{\sqrt{\chi(\chi-1)}} \operatorname{Arth} \sqrt{\frac{\chi-1}{\chi},}, \quad \chi \geq 1, \\ \frac{1}{\sqrt{\chi(1-\chi)}} \operatorname{arctg} \sqrt{\frac{1-\chi}{\chi}}, & \chi<1 .\end{cases}
$$

The RHIC ring consists mainly of regular FODO cells. Integrating Eq. (22) through FODO cells only, one obtains

$$
\left[\begin{array}{c}
\tau_{x} \\
\tau_{y} \\
\tau_{s}
\end{array}\right]_{\mathrm{Au}}=\left[\begin{array}{c}
-223 \\
-24.6 \\
+2.42
\end{array}\right] \min \text { and } \quad\left[\begin{array}{c}
\tau_{x} \\
\tau_{y} \\
\tau_{s}
\end{array}\right]_{\mathrm{p}}=\left[\begin{array}{c}
+905 \\
-2261 \\
+88.9
\end{array}\right] \mathrm{min}
$$

at the beginning of the injection. The growth times $\tau_{x, y, s}$ are defined as

$$
\tau_{x, y, s}=\left(\frac{1}{\sigma_{x, y, s}} \frac{\mathrm{d} \sigma_{x, y, s}}{\mathrm{~d} t}\right)^{-1} .
$$

After $2 \mathrm{~min}$ (gold ions) or $30 \mathrm{~s}$ (protons) one has

$$
\left[\begin{array}{c}
\frac{\Delta \sigma_{x}}{\sigma_{x}} \\
\frac{\Delta \sigma_{y}}{\sigma_{y}} \\
\frac{\Delta \sigma_{p}}{\sigma_{p}}
\end{array}\right]_{\mathrm{Au}, 2 \min }=\left[\begin{array}{l}
-0.01 \\
-0.10 \\
+1.03
\end{array}\right] \text { and }\left[\begin{array}{c}
\frac{\Delta \sigma_{x}}{\sigma_{x}} \\
\frac{\Delta \sigma_{y}}{\sigma_{y}} \\
\frac{\Delta \sigma_{p}}{\sigma_{p}}
\end{array}\right]_{\mathrm{p}, 30 \mathrm{~s}}=\left[\begin{array}{l}
+8 \cdot 10^{-4} \\
-8 \cdot 10^{-5} \\
+8 \cdot 10^{-3}
\end{array}\right] .
$$

This is certainly a conservative estimate since the emittances are already enlarged compared to the values given in Table I. The longitudinal emittance growth for gold ions is significant. However, it is interesting to plot the longitudinal emittance growth as a function of 
bunch length while keeping the longitudinal emittance constant. This is done in Figure 1. A shorter bunch would lead to less emittance growth. The rms bunch length is given by ${ }^{10}$

$$
\sigma_{s}=\left(\frac{A}{Z e} \frac{C^{2}|\eta| E \epsilon_{s}^{2}}{2 \pi h V_{\mathrm{RF}}}\right)^{1 / 4}
$$

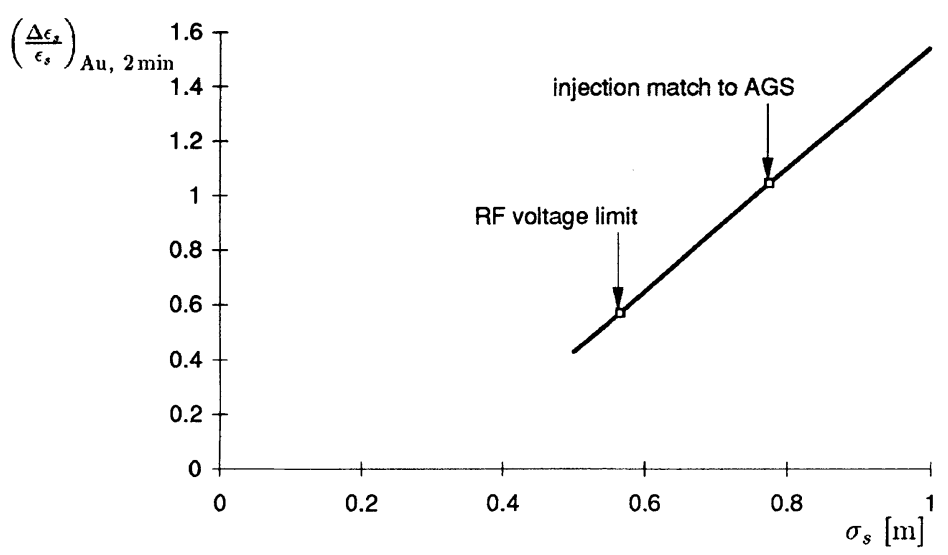

FIGURE 1 Longitudinal emittance growth of gold ions as a function of rms bunch length at injection. The longitudinal emittance is kept constant.

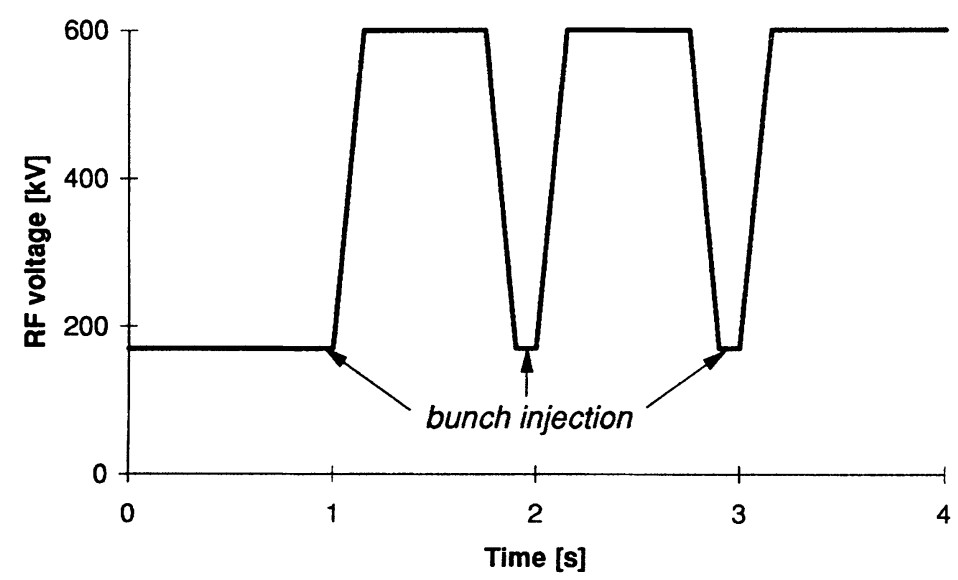

FIGURE 2 Injection scheme to reduce the space charge induced longitudinal emittance growth. 
TABLE IV Expected emittance growth in RHIC during injection. The longitudinal emittance growth of a gold ion beam due to intrabeam scattering given in brackets is the number for the injection scheme described in Section 5

\begin{tabular}{lcccccccc}
\hline Process & \multicolumn{3}{c}{$\mathrm{Au}^{79+}$} & & \multicolumn{3}{c}{$\mathrm{p}^{+}$} \\
\cline { 2 - 3 } & $\begin{array}{c}\Delta \epsilon_{x} / \epsilon_{x} \\
{[\%]}\end{array}$ & $\begin{array}{c}\Delta \epsilon_{y} / \epsilon_{y} \\
{[\%]}\end{array}$ & $\begin{array}{c}\Delta \epsilon_{s} / \epsilon_{s} \\
{[\%]}\end{array}$ & & $\begin{array}{c}\Delta \epsilon_{x} / \epsilon_{x} \\
{[\%]}\end{array}$ & $\begin{array}{c}\Delta \epsilon_{y} / \epsilon_{y} \\
{[\%]}\end{array}$ & $\begin{array}{c}\Delta \epsilon_{s} / \epsilon_{s} \\
{[\%]}\end{array}$ \\
\hline Steering error (no damper) & 48 & 68 & 4 & & 80 & 112 & 3 \\
Steering error (damper) & 10 & 10 & 0 & & 10 & 10 & 0 \\
Optical mismatch $(\alpha, \beta)$ & 2 & 4 & $2.5-10$ & & 2 & 4 & $2.5-10$ \\
Dispersion mismatch $\left(D, D^{\prime}\right)$ & 0.03 & 0.06 & - & & 0.03 & 0.06 & - \\
Intrabeam scattering & -1 & -10 & $103(57)$ & & 0 & -0.8 & 0.8 \\
\hline
\end{tabular}

and with the RF voltage available $(600 \mathrm{kV}$ maximum, $170 \mathrm{kV}$ are needed to match the AGS RF system at injection) the rms bunch length could be shortened adiabatically from 0.774 to $0.565 \mathrm{~mm}$ after injection of a new bunch (see Figure 1). For the injection of the next bunch (after $2 \mathrm{~s}$ ) the length would be increased again adiabatically to match the AGS RF system and so on (see Figure 2). With such a procedure the emittance growth could be restricted to $57 \%$ (compared to $103 \%$ without these changes).

\section{SUMMARY}

Transverse and longitudinal emittance growth due to steering errors and optical mismatch should not exceed $10 \%$ for protons and gold ions. Space charge does not contribute to emittance growth. However, a large longitudinal emittance growth for gold ion beams is expected from intrabeam scattering. This emittance growth can be reduced by decreasing the bunch length adiabatically during the time between the injection of consecutive bunches. These results are summarized in Table IV.

\section{Acknowledgements}

The authors are thankful to D.P. Deng and J. Rose for information about the RHIC RF system. 


\section{References}

[1] W. Fischer and T. Satogata, A simulation study on tune modulation effects in RHIC, BNL RHIC/AP/109 (1996).

[2] G.F. Dell and S. Peggs, Simulation of the space charge effect in RHIC, Proceedings of the 1995 IEEE Particle Accelerator Conference, Dallas (1995).

[3] S. Peggs and W.W. MacKay (Eds.), Collective Instabilities in RHIC, BNL RHIC/ AP/36 (1994); The conceptual design of the RHIC RF system, Internal report (1994); RHIC Design Manual, revised September 1995.

[4] P.J. Bryant, Beam transfer lines, CERN 94-01 (1994).

[5] D.A. Edwards and M.J. Syphers, An Introduction to the Physics of High Energy Accelerators (John Wiley \& Sons, 1993).

[6] T. Satogata et al. Physics of the AGS-to-RHIC transfer line commissioning, Proceedings of the 1996 European Particle Accelerator Conference, Barcelona, BNL RHIC/AP/104 (1996).

[7] A.W. Chao, Physics of Collective Beam Instabilities in High Energy Accelerators (John Wiley \& Sons, 1993).

[8]. M. Conte and W.W. MacKay, An Introduction to the Physics of Particle Accelerators (World Scientific, 1991).

[9] J. Wei, Evolution of hadron beams under intrabeam scattering, Proceedings of the 1993 IEEE Particle Accelerator Conference, Washington, D.C. (1993).

[10] S. Peggs and J. Wei, Longitudinal phase space parameters, BNL RHIC/AP/106 (1996). 